\title{
What Role Does Hydrological Science Play in the Age of Machine Learning?
}

Grey S. Nearing ${ }^{1}$, Frederik Kratzert ${ }^{2}$, Alden Keefe Sampson ${ }^{3}$, Craig S. Pelissier $^{4}$, Daniel Klotz ${ }^{2}$, Jonathan M. Frame ${ }^{1}$, Cristina Prieto ${ }^{5}$, Hoshin V. Gupta $^{6}$

\author{
${ }^{1}$ Department of Geological Sciences, University of Alabama; Tuscaloosa, AL USA \\ ${ }^{2}$ LIT AI Lab \& Institute for Machine Learning, Johannes Kepler University; Linz, Austria \\ ${ }^{3}$ Upstream Tech, Natel Energy Inc.; Alameda, CA USA \\ ${ }^{4}$ NASA Center for Climate Simulation, NASA Goddard Space Flight Center; Greenbelt, MD USA \\ ${ }^{5}$ IHCantabria - Instituto de Hidráulica Ambiental de la Universidad de Cantabria, Santander, Spain \\ ${ }^{6}$ Department of Hydrology and Atmospheric Sciences, University of Arizona; Tucson, AZ USA
}

\section{Key Points:}

- Hydrology lacks scale-relevant theories but deep learning experiments suggests that these theories should exist

- It is up to hydrologists to clearly show where and when hydrological theory adds value to simulation and forecasting

Corresponding author: Grey Nearing, gsnearing@ua.edu 


\begin{abstract}
This paper is derived from a keynote talk given at the Google's 2020 Flood Forecasting Meets Machine Learning Workshop. Recent experiments applying deep learning to rainfallrunoff simulation indicate that there is significantly more information in large-scale hydrological data sets than hydrologists have been able to translate into theory or models. While there is growing interest in machine learning in the hydrological sciences community, in many ways our community still holds deeply subjective and non-evidence-based preferences for models based on a certain type of 'process understanding' that has historically not translated into accurate theory, models, or predictions. This commentary is a call to action for the hydrology community to focus on developing a quantitative understanding of where and when hydrological process understanding is valuable in a modeling discipline increasingly dominated by machine learning. We offer some potential perspectives and preliminary examples about how this might be accomplished.
\end{abstract}

\title{
1 Beven's Clouds
}

On April 27, 1900 William Thomson (Lord Kelvin) gave his 'Two Clouds' speech ('Nineteenth-Century Clouds over the Dynamical Theory of Heat and Light') at the Royal Institution, in which he argued that "The beauty and clearness of the dynamical theory, which asserts heat and light to be modes of motion, is at present obscured by two clouds." The two open problems in physics that Kelvin referred to were the failure of the MichelsonMorley experiment to detect the luminous ether ("how could the earth move through an elastic solid, such as essentially is the luminiferous ether?"), and the ultraviolet paradox ("the Maxwell-Boltzmann doctrine regarding the partition of energy"). Within a decade, Einstein had proposed fundamentally novel insights that led to two paradigm shifts that define modern physics to this day - the transformation of these two 'clouds' into relativity and quantum mechanics.

In 1987, Keith Beven gave what might be considered hydrology's version of the Two Clouds speech at a symposium of the International Association of Hydrological Sciences (IAHS) (Beven, 1987). He took a perspective inspired by Thomas Kuhn's theory of scientific revolutions (Kuhn, 1962) to argue that "[t]he extension of laboratory scale theory to the catchment scale is unjustified and that a radical change in theoretical structure (a new paradigm) will be required before any major advance can be made in [predicting catchment-scale rainfall-runoff responses]." He proposed that two things would be necessary to push the field of surface hydrology into a new period of 'normal science': (i) scale-relevant theories of watersheds ("h/ydrology in the future will require a macroscale theory that deals explicitly with the problems posed by spatial integration of heterogeneous nonlinear interacting processes"), and (ii) uncertainty quantification ("[s]uch a theory will be inherently stochastic and will deal with the value of observations and qualitative knowledge in reducing predictive uncertainty.")

Unfortunately, hydrology has not had its Einstein (with all due respect to A. Einstein, 1926; H. A. Einstein, 1950). Nine decades from the establishment of the Hydrology section of the American Geophysical Union and after more than a half-century of computer-based hydrological modeling (Crawford \& Burges, 2004), Blöschl et al. (2019) listed as one of the twenty three 'Unsolved Problems in Hydrology': "what are the hydrologic laws at the catchment scale and how do they change with scale?'

\section{Tilting at Windmills}

There are several potential reasons why the search for scale-relevant theories in hydrology has been unsuccessful, but lack of effort is not one of them (e.g., Beven, 2006b; Blöschl \& Sivapalan, 1995; Dooge, 1986; Peters-Lidard et al., 2017; Sivapalan, 2006). One potential reason is simply that there might be no scale-relevant theories to find - it is 
possible that macroscale watershed behaviors are dominated by heterogeneity, meaning that there is little consistency across different basins. As summarized by Hrachowitz et al. (2013), "Beven (2000) highlighted the varying importance of different hydrological processes, active at different time scales in different catchments, and thereby emphasized uniqueness of place as a consequence of the variability of nature."

Alternatively, it could be the case that there are consistent macroscale patterns in hydrologic behaviors across watersheds, but we lack sufficient observations (type, scale, scope) to discover these similarities. Again, as summarized by Hrachowitz et al. (2013), "ii]t was realized that increased physical model realism (and complexity) requires both more input data and more model parameters, which are rarely available with sufficient detail to account for catchment heterogeneity at the required resolution."

Uniqueness of place and lack of data are, in our experience, two of the most common hypotheses about why hydrology lacks both scale-relevant theories of watersheds. The alternative to such hypotheses is that these theories could exist and that there is enough information in available observation data that we could have discovered them, but that hydrologists simply have failed to do so. Prior to last year, it is fair to say that as a community we did not know which of these reasons was the cause of our lack of success. However, with the accelerating development of modern machine learning (ML), and deep learning (DL) in particular, we know that the reason is the third one listed: watershedscale theories (and models) could have been derived from currently-available observation data, but the hydrology community simply failed to do so.

The reason that we know this is because general models can be learned with DL. In a large sample study using 30 years of data from several hundred basins in the continental United States, DL gave better daily streamflow predictions on average in ungauged basins than traditional hydrology models when calibrated to long data records in gauged basins (Kratzert, Klotz, Herrnegger, et al., 2019). That study used benchmarks based on (i) a modern process-based model that was the product of several millions of dollars of development funding, and (ii) a conceptual model calibrated separately for each individual basin (Figure 1). These DL models have been benchmarked against a number of conceptual and process models calibrated both locally and regionally using a variety of metrics and hydrological signatures (Kratzert, Klotz, Hochreiter, \& Nearing, 2020; Kratzert, Klotz, Shalev, et al., 2019). The fact that DL learned to predict in unseen basins better than traditional models in gauged basins indicates that there exists inter-basin consistency that we should be able to exploit and develop into a watershed-scale theory of rainfall-runoff behavior.

The problem of prediction in ungauged basins (PUB) (Hrachowitz et al., 2013; Sivapalan et al., 2003) is fundamentally a problem of extrapolation. Unlike both conceptual and process-based hydrology models, and also unlike shallow ML models that the hydrological science community has used in the past (e.g., Hsu, Gupta, \& Sorooshian, 1995), DL models work better when trained on multiple catchments than when trained on individual catchments (Figure 2; also see the more thorough data scaling analysis by Gauch, Mai, and Lin (2019)). This means that DL models learn relationships from a large sample of hydrological variability and are able to translate those learned relationships into better predictions in any individual basin. In contrast, traditional hydrology models are best when calibrated to individual basins, and performance always degrades when transferring to other basins or when using regional calibration.

It is often claimed that one of the reasons hydrology models don't extrapolate well is because they are over-calibrated. Hrachowitz et al. (2013) reported that "several authors (Kirchner, 2006; McDonnell et al., 2007; Wagener, Sivapalan, Troch, 83 Woods, 

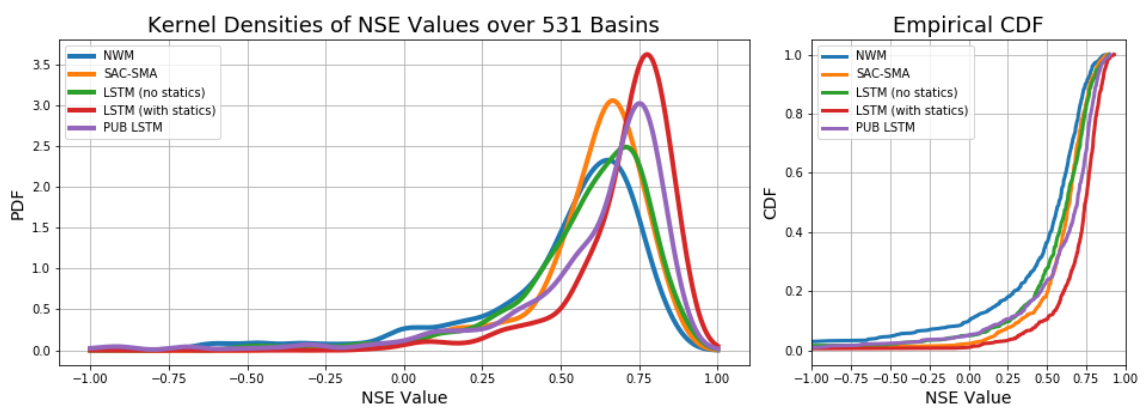

Figure 1. Results from Kratzert, Klotz, Herrnegger, et al. (2019) showing the empirical and cumulative distributions of model performance (Nash Sutcliffe Efficiencies) over a 15-year test period in 531 CAMELS catchments. SAC-SMA is the Sacramento Soil Moisture Accounting model, NWM is the National Water Model Reanalysis, and LSTMs are Long Short Term Memory networks (a type of deep learning architecture). The PUB-LSTM is the deep learning model applied in out-of-sample catchments. The other LSTM models (with and without statics) refer to deep learning models that were trained on all catchments (i.e., no out-of-sample catchments) and either did or did not use static catchment attributes (e.g., soils, vegetation, topography, etc.) as inputs.

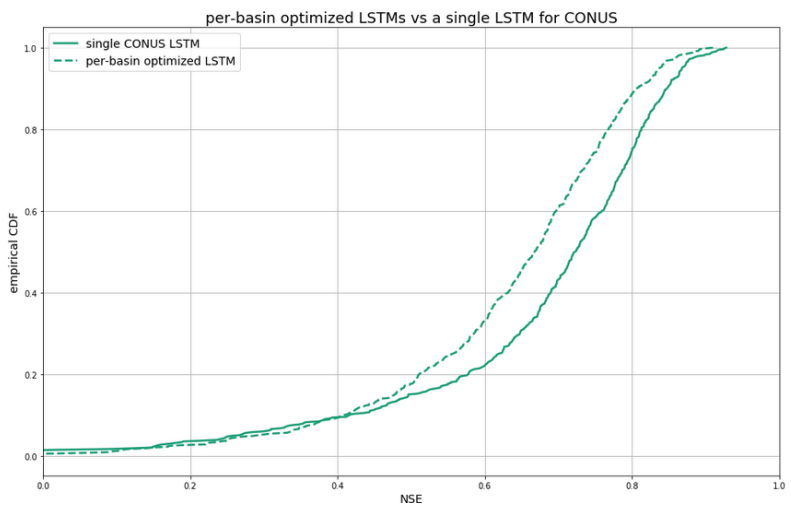

Figure 2. Cumulative distributions of NSE values over the same 531 CAMELS basins used by Kratzert, Klotz, Shalev, et al. (2019) from a single model trained over all basins (single CONUS LSTM) vs. separate models trained at each basin (per-basin optimized LSTM).

2007) ${ }^{1}$ expanded on and strongly reiterated Klemeš's (1986b) arguments that models which perform adequately well during calibration, but fail to predict the hydrological catchment response in validation, frequently do so because they do not sufficiently represent the realworld processes that control the catchment response. Rather, their often high number of parameters together with the limited number of constraints (including both calibration objectives and calibration criteria) resulted in high degrees of freedom, i.e. poorly conditioned parameter estimation problems, so that models behaved more like "mathematical marionettes." The problem is that this is not true. DL models generally have several orders of magnitude more degrees of freedom than calibrated conceptual models, and it is this lack of regularization that allows them to learn general and transferable hydro-

\footnotetext{
${ }^{1}$ These references are apparently incorrect in the quoted manuscript.
} 
logical relationships. Using DL as a benchmark demonstrates that it is the regularization in the traditional models (i.e., the hydrological theory that the model structures are based on) that is actually the cause of their lack of generality and transferability, rather than this being a problem of over-parameterization.

To summarize, this benchmarking between DL and traditional hydrology models demonstrates three things. First that hydrologists could have developed general, scalerelevant theories of watersheds from available data, but failed to do so. Second, that our understanding of why such theories don't exist were incorrect - neither uniqueness of place nor lack of data was a valid reason for this failure. Third, that our understanding of why our existing models perform poorly in extrapolation is also incorrect - this is not due to a lack of regularization or to over-parameterization, but instead due to bad theory - the regularization (structure) that does exist in these models actively hurts us.

\section{Black Swans and Black Boxes}

In the preceding section, we argued that DL experiments suggests that new watershedscale rainfall-runoff theory should exist, however DL models do not currently give us those theories. There are two ways we might think about this issue - both are currently open problems in hydrology.

First, we can leverage advances in explainable AI (XAI; Samek, 2019). It is often said that machine learning is a black box, and while there is some sense in which this is true, there is a much more important sense in which we should think about DL models as containing complex, multi-layered, structured information that is accessible to us if we choose to query it. Recognizing this, our job as scientists becomes a problem of translation: the information we want is in the models and we must learn how to translate that information into something that is human-interpretable.

Trained DL models typically don't yield new theory directly, however process-based models don't either. New insights from modeling studies come from probing models with various types of diagnostic tools (e.g., Martinez \& Gupta, 2010; Nearing, Ruddell, Clark, Nijssen, \& Peters-Lidard, 2018; Ruddell, Drewry, \& Nearing, 2019; Yilmaz, Gupta, \& Wagener, 2008), many of which are equally applicable to DL models. Examples of these tools are things like sensitivity analyses to understand (e.g.,spatiotemporal) input contributions (e.g., Sundararajan, Taly, \& Yan, 2017), counterfactuals to understand cause and effect, (e.g., Pearl, 2013; Ribeiro, Singh, \& Guestrin, 2016), or DL-specific tools like embedding layers and feature layer analyses (e.g., Bianchi, Rossiello, Costabello, Palmonari, \& Minervini, 2020; Q. Wang, Mao, Wang, \& Guo, 2017). We will give examples of hydrologicallyrelevant XAI in the context of the experiments described in Section 2 presently.

Second, we could use DL model for hypothesis testing. Instead of extracting information from trained DL models, we can put hydrological theory into these models and assess improvement (or otherwise). From an ML perspective this is a regularization problem, and common methods include things like (i) regularizing the loss function to penalize violations of physical principles like conservation, monotonicity, etc. (e.g., Nabian \& Meidani, 2020), (ii) augmenting scientific models with DL structures (e.g., Pelissier, Frame, \& Nearing, 2020; Rackauckas et al., 2020) and (iii) architecturally constrained neural networks (e.g., Beucler et al., 2019; Daw et al., 2020).

According to most interpretations of the scientific method, hypotheses are tested by comparing predictions with observations. The results discussed in Section 2 can be interpreted as a hypothesis test that compares the information content of hydrological theory as encoded into models relative to a null hypothesis derived from data (Nearing, Ruddell, Bennett, Prieto, \& Gupta, 2020). This does not mean that all hydrological theory encoded in those models should be rejected, but it is a challenge to disaggregate the good parts of that body of theory - that may provide significant hydrological informa- 
tion - from the bad (H. V. Gupta, Wagener, \& Liu, 2008). This is a classic problem of underdetermination (Laudan, 1990). In this case, the problem is due in part to the fact that for complex systems like watersheds it is necessary to aggregate a large number of different theories and bridge principles (Nagel, 1961) into predictive models. From a philosophical (and completely untested) perspective, we suggest that DL might help with the underdetermination problem to some extent by providing a modeling framework that allows us to aggregate pieces of hydrological theory into a functional, integrated model without requiring that the model includes theories of everything. For example, we might test hypotheses about conservation at various scales (or closure in data) without requiring an explicit assumption about infiltration, evapotranspiration, or groundwater simply by embedding conservation laws into DL models (e.g., see Section 7.2). This is not possible with purely process-driven models that need descriptions of everything in order to account for every relevant catchment process. DL can learn the basic functional relationships from data and we can, in principle, assess the information content of any particular hypothesis by adding that hypothesis as a constraint on the DL model. While this paper was in review, an excellent example of this was provided by (Jiang, Zheng, \& Solomatine, 2020), who included process modules as layers in deep tensor networks.

An example of the looking for explainability in a trained model is in Fig. 3. This figure shows the sensitivity of a time series DL model to past inputs. The model learned to store winter precipitation and release this as runoff when temperature and radiation increased in the spring. (Kratzert, Herrnegger, Klotz, Hochreiter, \& Klambauer, 2019) showed that a DL time series model trained with inputs of precipitation and daily air temperature and targets of only daily streamflow contained internal states that correlated with snow cover and soil water storage. They showed that these 'snow' states were sensitive to inputs only when temperatures were below zero. None of this behavior was prescribed a priori - the model learned hydrologically-relevant, interpretable behavior about latent (unobserved) variables.

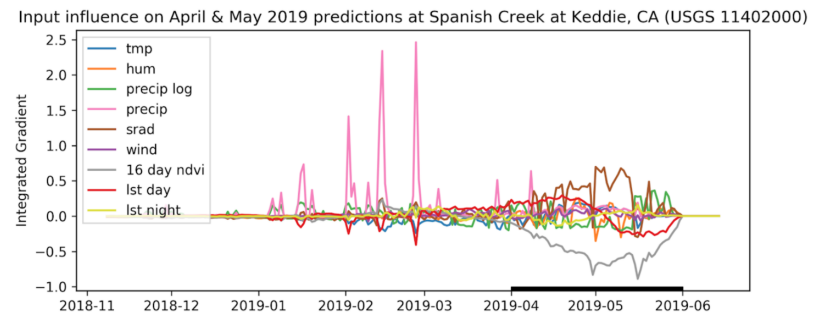

Figure 3. Sensitivity analysis using integrated gradients (Sundararajan et al., 2017) that shows the relative contributions to simulated streamflow during the months of April-May (heavy black shading on the x-axis) from the time-series of past inputs. The DL model learns to store winter precipitation and responds to increasing temperature and solar radiation in the spring.

Looking at the transferability and catchment similarity issues discussed in Section 2, Kratzert, Klotz, Shalev, et al. (2019) constructed a DL network with an embedded feature layer that quantified catchment similarity along a number of learned dimensions (Figure 4). The features extracted from the trained network represent how the DL model transformed observable catchment characteristics into a representation of similarity and diversity in rainfall-runoff relationships. This matrix looks a little like noise, but it is a better representation of catchment similarity than anything human scientists have so far been able to develop. If we want to understand the information encoded in this matrix, then the job ahead of us is to translate this information into a human-interpretable form. Kratzert, Klotz, Shalev, et al. (2019) used dimensionality reduction to relate first-order 
features in this similarity matrix with observable catchment characteristics and found that vegetation type and seasonality were the dominant influences.

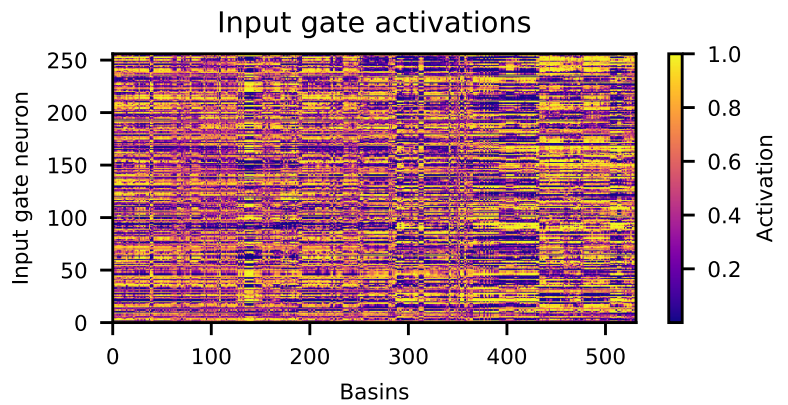

Figure 4. Results from Kratzert, Klotz, Shalev, et al. (2019) showing a matrix representing catchment similarity as identified by a deep learning model. There are 531 catchments (x-axis) and 256 model states (y-axis). Each state is activated for any individual catchment to some degree in the range $[0,1]$, with 0 meaning that the state is not used for that particular catchment. Similar catchments share more of this state space and dissimilar catchments share less.

While ML has been used in hydrology for decades, the ability (at least partially due to computational advances) to arrange shallow learning models into complex structures with feature layers that can learn multi-scale patterns opens the door to leveraging diverse (e.g., multi-catchment) data in interpretable ways. The idea that ML models are 'black boxes' is more of a testament to a lack of inspection, rather than to a fundamental limitation of the models themselves. It's worth noting that the DL models used by Kratzert, Klotz, Shalev, et al. (2019) were invented around the same time (Hochreiter, 1991; Hochreiter \& Schmidhuber, 1997) as some of the earliest shallow neural network applications in hydrology (e.g., Hsu et al., 1995). As a discipline, we have not done a great job of keeping pace with developments in ML.

\section{Known Unknowns}

The second 'cloud' in Beven's (1987) speech was uncertainty. There has been an enormous amount of attention paid to this topic in the hydrological sciences (e.g., Beven, 2006a, 2009, 2016; Beven \& Binley, 2014; Beven, Smith, \& Freer, 2007; Beven, Smith, Westerberg, \& Freer, 2012; Beven, Smith, \& Wood, 2011; Beven, Smith, \& Freer, 2008; Clark, Kavetski, \& Fenicia, 2011; P. Kumar, 2011; Mantovan \& Todini, 2006; Montanari, 2007; Montanari \& Koutsoyiannis, 2012; Nearing, 2014; Nearing \& Gupta, 2018; Pappenberger \& Beven, 2006; Renard, Kavetski, Kuczera, Thyer, \& Franks, 2010; Stedinger, Vogel, Lee, \& Batchelder, 2008; Todini \& Mantovan, 2007; Vrugt, Ter Braak, Gupta, \& Robinson, 2009), however we have not had a major breakthrough that led to a paradigm shift. We've suggested previously (Nearing, Tian, et al., 2016) that the uncertainty literature in hydrology is somewhat detached from the discussion about uncertainty that is taking place in the larger academic (science and philosophy) communities. However, irrespective of that opinion, our community has not developed the stochastic theory of watersheds that Beven (1987) anticipated.

Dooge (1986) offered a discussion about why finding scale-relevant laws is difficult in many branches of science. His argument was that there are two basic categories of scientific theory: mechanistic and aggregate. In the former - mechanistic theories - we track properties (e.g., position, velocity) of individual components of a system, and the result- 
ing model is usually expressed as a system of partial differential equations (PDEs). In the latter - aggregate theories - we rely on ergodic properties like the law of large numbers to derive consistent statistical approximations (e.g., temperature, density) at scales that are much larger than the individual components of a system. The prototypical example of a mechanistic-type theory are Newton's laws, and the prototypical example of an aggregate-type theory is thermodynamics. Dooge borrowed the image in Figure 5 from Weinberg (1975) to illustrate this dichotomy - watersheds live in the middle area of organized complexity, where complexity (heterogeneity) is at a similar scale to randomness (lack of information).
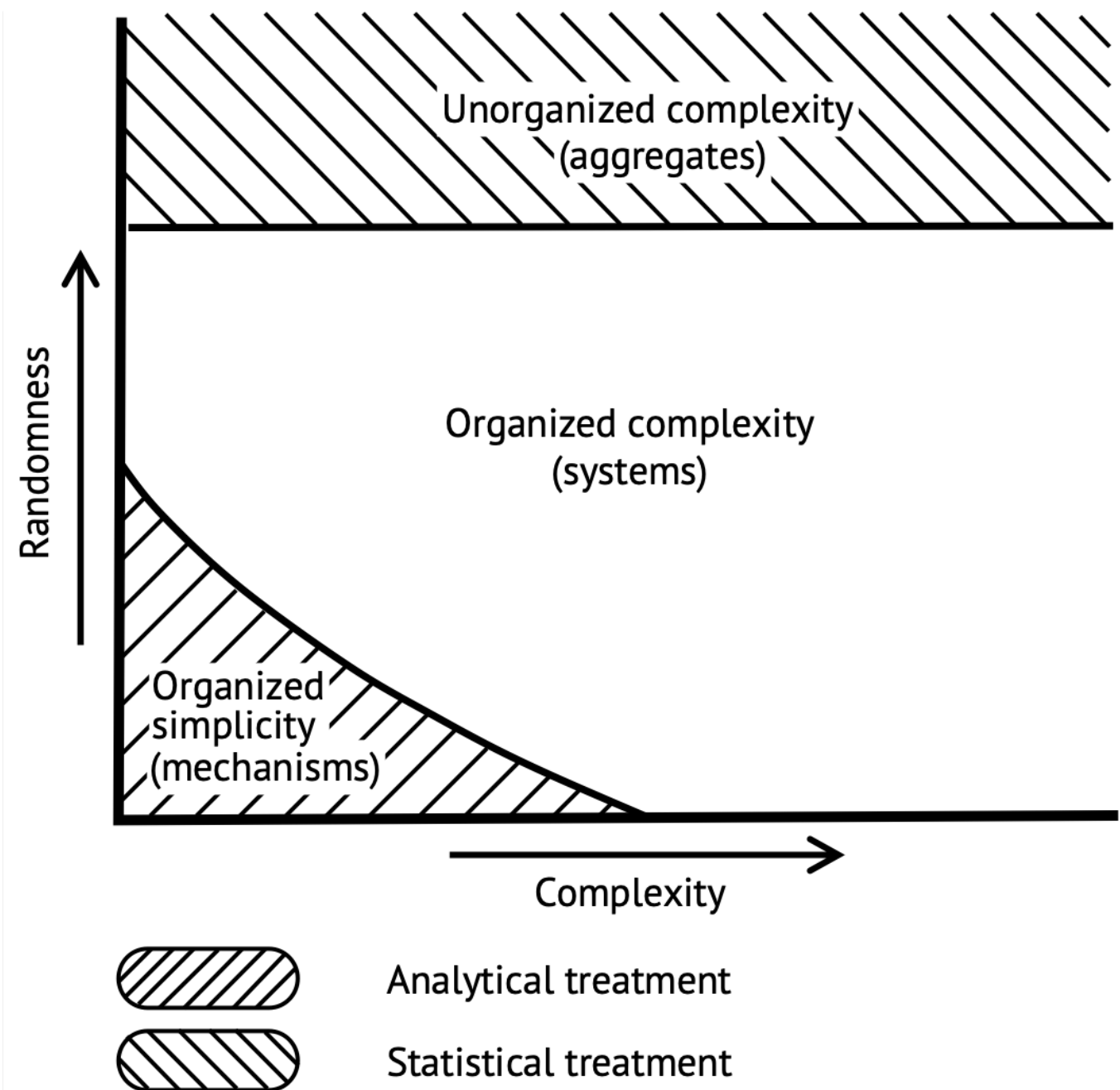

Figure 5. Recreation of an illustration that Dooge (1986) borrowed from Weinberg (1975) to show different types of successful theories in science. Watersheds arguably live in the area of organized complexity, where the complexity (heterogeneity) is at a similar scale to the randomness (lack of information).

Beven imagined a hydrological theory that is fundamentally stochastic to account for heterogeneity. This is different than how hydrologists currently treat uncertainty. Typical modeling approaches are mechanistic and treat a lack of complete information by adding additional (usually probabilistic) structure to a modeling problem. What we mean 
by this is that our basic hydrologic theories are largely deterministic, and we represent lack of complete information by adding distributions on top of model inputs, structures, and predictions. Intuitively, it seems odd that we add more structure to a problem to represent a lack of information. Beven's (1987) view of hydrologic theory is compelling in the sense that it would be preferable to have a theory of watersheds that is itself an aggregate-type theory, since at least a significant portion of the variability and complexity in watershed behaviors are due to both landscape and process heterogeneity.

Machine learning offers something like this in a straightforward way. Instead of predicting the quantities of interest directly, we can predict distributional representations (e.g., probabilistic, fuzzy, etc.) directly from input data. This can be as simple as having the output of a DL model be the parameters of a parametric distribution (e.g., a mixture density, Bishop, 1994), or the quantiles of a nonparametric distribution (Taylor, 2000). An example of this is shown in Figure 6, which shows the weights of a mixture density over streamflow predicted by a DL model. The training loss function in this case was a likelihood function, and the model did not learn the mixture density parameters directly, instead it learned how to predict these parameters from dynamic inputs. This figure shows that the individual kernels of the mixture density respond in hydrologicallyrelevant ways - for example, some of the mixture weights have a seasonal cycle, and some are active only in rising or falling limbs of the hydrograph. It is important to understand that the DL model here maps directly from inputs (atmospheric forcings and static basin attributes; Addor, Newman, Mizukami, \& Clark, 2017) to predicted probabilities, rather than sampling a priori probabilities over different model components. There is no need to prescribe any a priori probabilities.

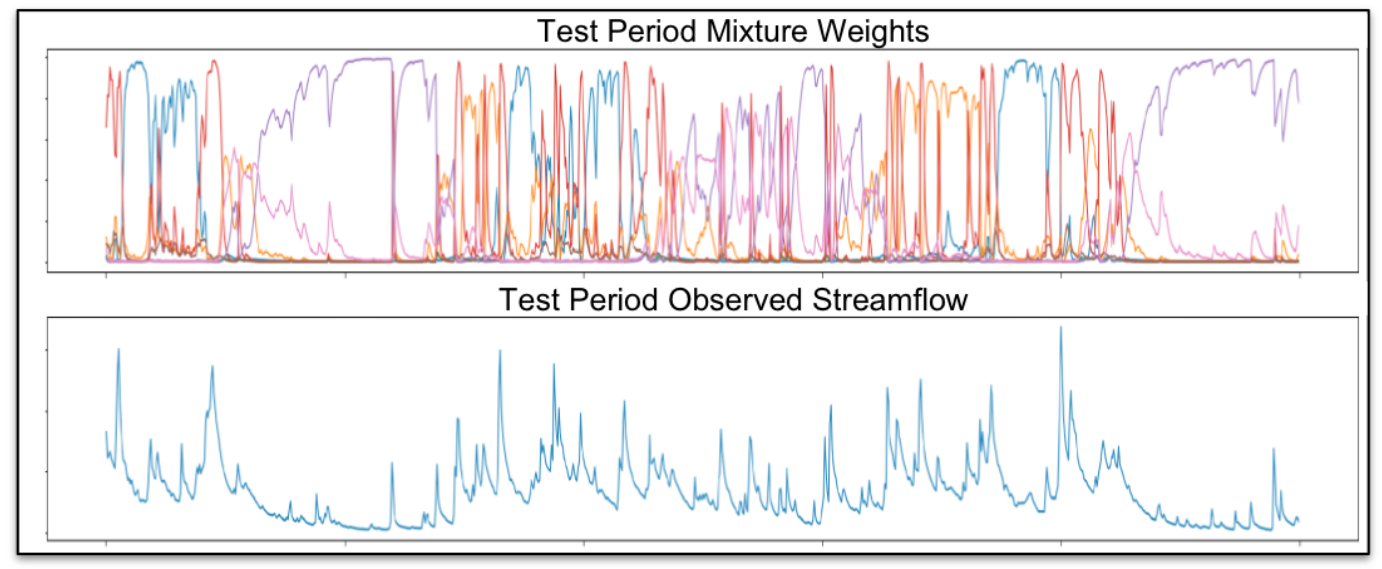

Figure 6. Mixture density weights (6 kernels) predicted by a deep learning model (top) as compared with the corresponding observed hydrograph (bottom). The mixture density weights vary in hydrologically-relevant ways - i.e., as a function of peaks (red) and recessions (blue).

We find an important distinction between generative vs. discriminative models (Nearing, Gupta, \& Crow, 2013). Generative models produce a joint distribution between targets, $Y$, and inputs, $X$, and then invert that distribution to obtain conditional predictive probabilities $p(Y \mid X)$. Discriminative models, on the other hand, map directly onto conditional probabilities. Discriminative models avoid the need to assign any a priori probabilities, and if we believe that we have some information about uncertainties associated with various inputs, these uncertainties can always be used as additional inputs into the model. 
Traditional hydrology models, on the other hand, are generative. We must first define all input distributions and our predicted distributions come from sampling those a priori prescribed distributions. When we use an ensemble to represent uncertainty, for example, the hydrological model or family of models produces a joint distribution between inputs and targets. Although we can sample the predictive conditional by simply looking at one ensemble member, the distribution itself does not exist except as implied by the ensemble where each ensemble member is a joint sample of $(X, Y)$. The bottom line is that in a generative approach, the predicted probabilities are defined in advance by the input or sampling probabilities.

While aggregate theories exist for certain hydrological fluxes (e.g., Singh, Yang, \& Deng, 2003; J. Wang \& Bras, 2011), most operational models are based on mechanistic theories - hydrologists have not developed an aggregate theory of watersheds. ML does not produce aggregate theories, but it does allow for discriminative modeling.

In addition to predicting probabilities directly, discriminative ML models can take any type of input, given sufficient training data. This offers an alternative to inverse methods like data assimilation for integrating ancillary data streams (Nearing et al., 2013). Feng, Fang, and Shen (2019), for example, used the discriminative approach to integrate lagged streamflow values in a (deterministic) DL streamflow model. In principle, it is feasible to add any type of input into one of these models as long as there is sufficient training data. We no longer need to prescribe the various input distributions directly - instead these are learned (either implicitly or explicitly) by the DL model from all available data in a way that is dynamic (i.e., changes) in time and place, and under different hydrologic conditions.

\section{Overlapping Magisteria: Faith and Fact in Hydrology}

In the previous sections, we motivated several arguments highlighting conceptual deficiencies in hydrological science that were demonstrated by recent findings from benchmarking DL models. This type of benchmarking result is not new - hydrologists have been testing ML models against both calibrated conceptual models and process-based models for at least a quarter century (Hsu et al., 1995, depending on how we define ML), and it has always been the case that ML generally outperforms other types of models (e.g., Abramowitz, 2005; Best et al., 2015; Nearing, 2013; Nearing, Mocko, Peters-Lidard, Kumar, \& Xia, 2016).

Todini (2007) framed the issue like this: "physical process-oriented modellers have no confidence in the capabilities of data-driven models' outputs with their heavy dependence on training sets, while the more system engineering-oriented modellers claim that data-driven models produce better forecasts than complex physically-based models." The key phrases in this sentence are 'confidence in' and 'better forecasts' - one is a statement of belief and one is a statement of fact.

Hydrology as an applied science is motivated by both epistêmê and technê (Parry, 2003). On one hand (technê), we are often funded to tackle acute societal needs for managing water resources and and mitigating water-related hazards. On the other hand (epistêmê), many of us are true curiosity-driven scientists and care fundamentally about increasing our understanding of the world around us. These two objectives, however, cannot be cleanly separated. Whether any individual hydrologist is personally motivated by societal relevance vs. primal curiosity (the analogy we want the reader to draw is with Gould's (1999) claim that "science treats factual reality, while religion treats human morality"), - the fact is that scientific hypotheses are tested by their ability to make accurate predictions. If our hypotheses do not translate into consistent accurate predictions, then they have not passed the basic test of science. The situation is a little more complicated when comparing the information content of data-driven vs. theory-driven models, since imperfect 
or incomplete theory can still be valid and useful, but hypotheses only become part of a body of theory if they translate into consistently accurate predictions.

The trend in the hydrology community has been toward more detailed process-based models based on essentially old theories of closure. As an example, Wood et al. (2011) suggested that "developing a hyperresolution hydrological prediction capability is a "grand challenge for hydrology" because of the significant modeling, computational, and data needs that will be required for global or continental predictions at these spatial resolutions [ $100 \mathrm{~m}$.' ') This was cited as a major part of the motivation for developing the US National Water Model (Salas et al., 2018), which doesn't out-perform simpler modeling strategies (e.g., Figure 1). Is the idea that if we keep increasing resolution and complexity, our models will reach a tipping point or there will be a step change in accuracy? Are we looking for incremental improvements with a trajectory sufficient to catch up to the accuracy we get from ML, even as the pace of development in basic ML and AI science increases and the Earth-observation record available for training continues to grow? Is this a reasonable expectation that more of the same will help solve the fundamental problem (lack of scale-relevant theory)?

In their report of the IAHS community-wide effort to outline key 'Unsolved Problems in Hydrology' (UPH), Blöschl et al. (2019) said that "[m]ost hydrologists would probably agree that [extrapolating to changing conditions] will require a more process-based rather than a calibration-based approach as calibrated conceptual models do not usually extrapolate well." Similarly, in a summary of a recent workshop on 'Big Data and the Earth Sciences' Sellars (2018) reported that "[m]any participants who have worked in modeling physical-based systems continue to raise caution about the lack of physical understanding of $M L$ methods that rely on data-driven approaches." The problem with these types of opinions is that in any case where we have sufficient observation data to benchmark models, ML does better, even out-of-sample (see references above). Similarly, Kirchner (2006) claimed that "ii]t is almost axiomatic that we need "physically based" models in order to make reliable predictions beyond the range of prior observations." This is not an axiom of any theorem or any tautology, it is a hypothesis at best, and one that has failed every empirical test put to it that we are aware of. This is not science, it is religion.

The reason that DL in particular has at least the potential to remain reliable under changing conditions is because these models can be trained on a large diversity of data. As any particular catchment changes, it is likely that there are other catchments in a global data set that is similar along one or more of the changing dimensions. There will always be some catchments that evolve outside of the training envelope in terms of climate change or other anthropogenic influences, and it is unknown how model (of any type) will behave in such situations. But most catchments in the world will have some analogue along most dimensions of climate or land use, etc. It will be a critical project to understand how to structure the right mix of theory and data for developing reliable models at, for example, the climate scale, but the presumption that such projections must be "physically-based" seems strange. Why would we ever prefer a model that does worse on the data that we actually have in-hand?

\section{Hydrology Beyond Streamflow}

The hydrological sciences are diverse and the discussion so far has been about catchment hydrology and streamflow. Supposing the reader accepts the arguments we've laid out so far, it's worth asking whether there are implications for other branches of the discipline. The answer is - of course - that we don't know. On one hand, there are major differences between the challenges faced in catchment hydrology vs. groundwater or ecohydrology or hydrometeorology, but at the same time it is difficult to overestimate the impact of DL and AI throughout all types of human endeavors. In hydrometeorology sev- 
eral studies have shown that even very simple regression models produce better estimates of radiation partitioning than process-based land surface models Abramowitz (2005); Best et al. (2015); Nearing, Ruddell, et al. (2018). Fang and Shen (2020) showed that DL can produce highly accurate soil moisture forecasts with remote sensing. Hydrometeorology is similar to streamflow hydrology in that observations are (relatively) abundant from satellites and mature sensor networks like FluxNet, etc. These fields are also similar in that the major sources of uncertainty are due to spatial heterogeneity at intermediate scales.

In groundwater, which is often more data limited than surface hydrology, many of the standard methods have close or direct analogs in ML already (e.g., Kriging is just Gaussian process regression Williams and Rasmussen (2006)). It may be the case that there is less potential for a fundamentally new result. One recent study reported that a physically-based groundwater model outperformed several shallow ML models (Chen, He, Zhou, Xue, \& Zhu, 2020). There have been some relatively small DL studies in groundwater hydrology (e.g., Mo, Zabaras, Shi, \& Wu, 2019; Sahoo, Russo, Elliott, \& Foster, 2017) that did not report transformative results.

It is hard to draw strong conclusions from the existing body of work. In all of these studies (including those by the current authors but with the notable exception of Fang, Pan, and Shen (2018)) is a lack of big data. ML does not have the ability to learn multiscale heirarchical patterns in the same way as DL, and therefore cannot leverage diversity in big data in the same way. After testing several shallow ML models, Chen et al. (2020) concluded that "the generalization ability of numerical model is superior to the machine learning models because of the inclusion of physical mechanism."

The basic problem is a lack of real investment into this type of effort. There are major programs across hydrologic disciplines to build comprehensive multi-scale models (e.g., groundwater (de Graaf, Condon, \& Maxwell, 2020), streamflow Li et al. (2015); Lin et al. (2019), hydrometeorology, (Rodell et al., 2004), and many others) but to our knowledge there is no similar effort to build global AI models. DL does not scale like traditional models - it works differently on large data sets than small data sets, - so small pilot studies do not tell us much.

There is no question that we are in a new information age, and that modern data science techniques have been transformative across scientific disciplines. The message that we would like to leave the reader with is that hydrologists currently don't know what how transformative this technology will across our discipline. We do not know this because we have not made a serious investment in AI-based hydrology. Our major modeling centers continue to invest primarily in old technologies and old approaches. In the case of streamflow hydrology, this has been a disaster. The point of this opinion piece is that there are clues that maybe the balance of data and theory will not look like what hydrologists anticipate (e.g., references in Section 5).

\section{Where the Sidewalk Ends}

So what could we do about this? The following subsections outline what we see as both immediate needs for expanding DL in hydrology, as well as some ideas about what the longer-term future could look like.

\subsection{Distributed Modeling}

The first immediate need is for spatiotemporal DL models in all areas of hydrology. We simply just need to make serious investments across the discipline to gather the data that each community has - across regions and countries, to the extent possible - and make a serious attempt to develop state-of-the-art AI models. 
We expect that first-order attempts at this type of project will look similar to current models with some explicit spatiotemporal extent/resolution and some number of latent (hidden) variables. Previously, we criticised calls for hyper-resolution modeling, and while the race to higher-density, more-of-the-same type models does seems to be a particularly unthoughtful idea, it is nevertheless the case that hydrological processes have both spatial and temporal components. We expect that within the next 1-2 years the community will develop several distributed DL watershed models (e.g., Moshe et al., 2020).

There are various ways that we might incorporate a multitude of different types of spatiotemporal data into trained models. DL allows for complex interactions between different feature layers, and fine tuning allows modelers to train individual components of a model. We can imagine a model developed by training different feature layers - perhaps themselves multi-layer DL models - and piecing these together to represent theoryguided architectures. As an example, we could imagine training a convolutional network to map from remote sensing data like SMAP (Entekhabi et al., 2010) to root-zone soil moisture by training directly on target data from in situ networks like the USDA Soil Climate Analysis Network (Schaefer, Cosh, \& Jackson, 2007) and/or FluxNet (Baldocchi et al., 2001). The weights of this trained convolutional layer(s) could then be frozen, and the trained network then used as one (of many) input feature layer(s) into an LSTM (or other time series model) for predicting streamflow (or evapotranspiration or groundwater recharge). In principle, input data streams could be integrated at arbitrary spatiotemporal resolutions so that irregular convolutional networks (e.g., graph convolutions) could be used for routing.

The details of this type of model will need to be worked out, but the potential for, and basic components and principles of, a DL-based integrated hydrology model are relatively clear. There is no fundamental limitation that precludes developing integrated DL hydrology models at multiple temporal and spatial scales. The questions that we anticipate are about what value will come from integrating different types of features and feature layers, and about how we might pre-train various feature layers to account for different types and scales of observational data in large, integrated models.

\subsection{Theory-Informed Machine Learning}

As mentioned in Section 5, there is a feeling among hydrologists and Earth scientists that models without explicit process representation might be unreliable under changing conditions. Although we don't know if this is really true, one way to approach this is to integrate physical constraints or process-based theory into DL models. The goal is to extract as much information as possible from a combination of theory and data. This is not a new idea - Karpatne et al. (2017) called for theory-guided data science, which consists of efforts to integrate scientific consistency into generalizable models. Notably, members of that same group later collaborated on development of a DL model that is architecturally constrained to not violate prescribed monotonicity relationships (Daw et al., 2019).

A simple and general way to enforce conservation constraints (e.g., mass, energy, momentum) in a DL architecture is to L1-normalize a set of bounded $(\in[0,1])$ activation functions, and scale by the conserved quantity. This concept can be integrated into almost any type of neural network architecture, including into the long short term memory networks used by Kratzert, Klotz, Herrnegger, et al. (2019) and Kratzert, Klotz, Shalev, et al. (2019). This concept is illustrated in Figure 7, and the result is a model that learns nonlinear input-state-output relationships that obey arbitrary and interacting conservation principles.

Another approach for directly combining process understanding with ML is to incorporate the ML models inside of a dynamical systems model. A basic approach was outlined by Ghahramani and Roweis (1999), where - effectively - an empirical model is 


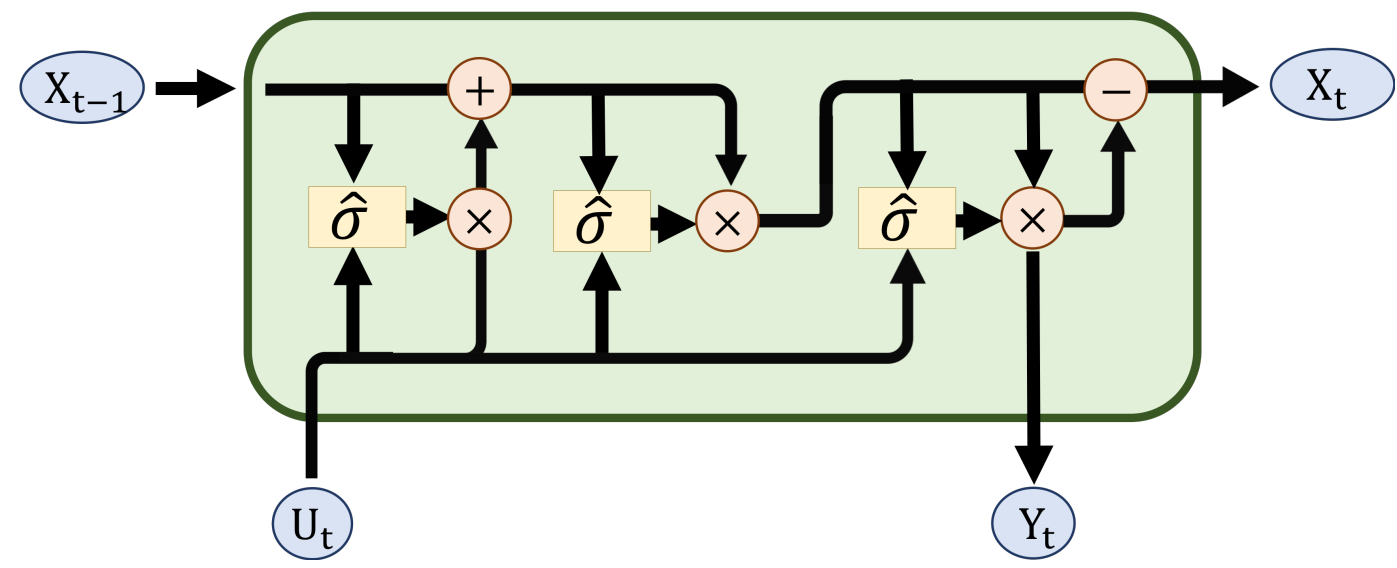

Figure 7. A time-recurrent deep learning network that is architecturally constrained to conserve mass, energy, and/or momentum. $U_{t}$ are time-dependent inputs, $Y_{t}$ are time-dependent outputs, and $X_{t}$ is a vector of $\mathrm{N}$ memory states in the network, $\hat{\sigma}$ represents a set of $N$ L1normalized sigmoid activation functions that produce a set of real values in $[0,1]$ that sum to unity. These are scaled by the conserved quantities (in the inputs and states) so that the total sum of the time-history of inputs plus outputs is always equal to the total sum of the system state. There are three sets of 'gates' in this network - an input gate that moves mass (energy, momentum) from inputs to states, a reshuffling gate that moves mass (energy, momentum) between states during each individual timestep, and an output gate that moves mass (energy, momentum) from states to outputs at each timestep.

trained on the analysis states resulting from data assimilation (e.g., by a Kalman-type filter). We can generalize this idea as follows:

Suppose that we have a dynamical systems model that solves a set of PDEs:

$$
\frac{d X}{d t}=f(X, U, \theta)
$$

where $X$ are modeled system states, $U$ are time-dependent boundary conditions, $\theta$ are model parameters, and function $f(\cdot)$ is the total divergence (inputs less outputs). A discretetime approximate solution might then be:

$$
X_{t}=f^{*}\left(X_{t-1}, U_{t}, \theta\right) .
$$

We can augment the $f^{*}(\cdot)$ state-transition function with a learned component, $g^{*}(\cdot)$, as:

$$
X_{t}=f^{*}\left(X_{t-1}, U_{t}, \theta\right)+g^{*}\left(X_{t-1}, U_{t}, \theta\right) .
$$

where $g^{*}(\cdot)$ is any ML model. As above, $g^{*}(\cdot)$ can itself be probabilistic so that equation 3 is a discrete-time solution to a set of stochastic PDEs. The challenge is to learn the $g^{*}(\cdot)$ function given that we can't expect to have direct observation pairs $\left(X_{t}, X_{t-1}\right)$ of all system states to use for supervised learning. As an example, Nearing and Gupta (2015) applied the data assimilation approach by Ghahramani and Roweis (1999) to the HyMod conceptual rainfall runoff model, and Pelissier et al. (2020) applied a similar technique to the Noah-MP land surface model for soil moisture accounting.

Another example of potential for theory-guided data science in hydrological workflows is for data assimilation itself. Significant information loss often results from assigning the distributions and parameters of a probability-based assimilation algorithm (Nearing, Yatheendradas, et al., 2018) and many assimilation algorithms require that the model 
and observation be in the same climatology (S. V. Kumar et al., 2012), meaning that these algorithms only treat stochastic error. One potential way to mitigate these problems is to use ML to learn relationships between model states and assimilated observations (e.g., Kolassa et al., 2018). As an example of this, Nearing (2013) derived the fixedform of the Kalman-type gain and its associated adjoint that results from assimilating with a Gaussian process observation operator.

We see theory-guided data science, and more specifically, physics-informed ML, as a likely strategy for simultaneously leveraging what we do know from scientific theory about catchment behavior with the now-undeniable ability of DL for extracting patterns and information directly from data. There is some indication that this might be useful: Figure 8 shows a comparison between the performance of a DL model applied to CAMELS basins vs. a calibrated conceptual model. This data is from Kratzert, Klotz, Herrnegger, et al. (2019), and the takeaway message is that while the DL model is better overall, it is not better everywhere. Kratzert, Klotz, Herrnegger, et al. (2019) could not find any relationship(s) between observable catchment characteristics and the difference in performance between these two models, but it is nevertheless apparent that there is at least the potential to improve by adding some elements of hydrologic theory to the DL architecture.
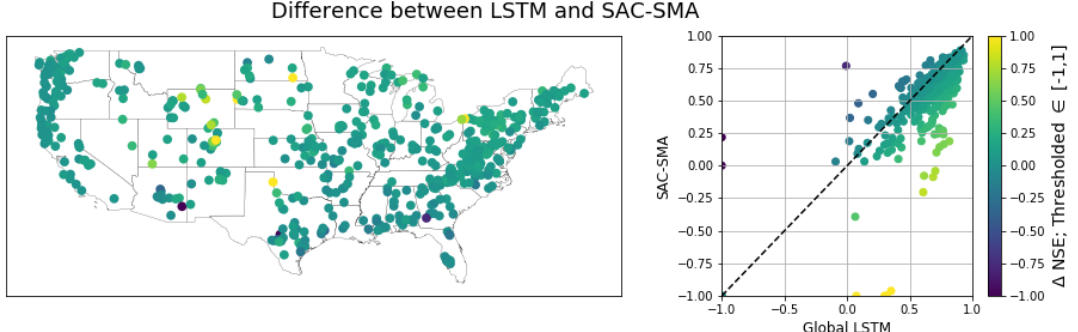

Figure 8. An illustration from Kratzert, Klotz, Herrnegger, et al. (2019) that compares a deep learning model (LSTM) against a calibrate conceptual model (SAC-SMA) over 531 CAMELS basins. The deep learning model does better on average, but not in every catchment, indicating that there is at least potential to improve by incorporating some of the conceptual constraints from SAC-SMA.

\subsection{Skip the Hydrologist}

Clark et al. (2016) gave an account of the sources of uncertainty (information loss) in a hydrological modeling chain. These are things like uncertainty in meteorological forcings from global circulation models (GCMs), downscaling forcings to the watershed scale, errors in the hydrological model structure, parameter uncertainty, etc. Each of these represents a step in a chain of information from the GCM dynamical core (i.e., Navier-Stokes approximations and data assimilation) to streamflow or other hydrological variables. Every step in this modeling chain introduces uncertainty. DL has the potential to let us skip at least several steps in this type of modeling chain by developing relationships directly between high-quality data sources.

Take as an example the largest source of hydrological error, which is typically precipitation data. This is true whether we are using the output of weather or climate models, interpolated gauge data, or remote sensing data from radar and/or satellites. The problem is exacerbated by downscaling. The major precipitation-related uncertainty in a global circulation model is due to parameterization of sub-grid cloud formation processes. There have been recent successes using ML to parameterize cloud physics and 
cloud formation (e.g., Gentine, Pritchard, Rasp, Reinaudi, \& Yacalis, 2018), which could help mitigate these issues to some extent, but we still have to feed these uncertain precipitation fields into a hydrology model that is subject to both parameter and structural uncertainties.

We could think about the problem in a different way. The four-dimensional pressure, wind, and temperature fields that result from Euler solutions in the dynamical cores of GCMs are relatively accurate, at least as compared with the accuracy of parameterized precipitation fields. We could, in principle, use DL to extract information directly from states of the dynamical core about terrestrial hydrological variables. For example, we could in principle develop four-dimensional convolutions to regress directly from GCM fields and digital elevation maps to pixel classifiers over satellite-derived maps of flood inundation, and thereby skip sources of information loss from (i) sub-grid convection parameterizations, (ii) GCM downscaling, (ii) lack of scale-relevant theories of watersheds, (iii) parameter equifinality, (iv) rating curves, etc. It is possible (perhaps likely) that this type of model would give more accurate inundation forecasts at similar lead times relative to state-of-the-art hydrology models, since this would skip uncertainties related to cloud physics parameterizations, downscaling, watershed parameterizations, etc. All of these things could be learned implicitly by a DL model.

The point is that DL offers at least the potential to make societally-relevant hydrological forecasts without any type of hydrological model or hydrological process understanding at all. Because DL allows for almost arbitrarily complex relationships, and has demonstrated to extrapolate well out-of-sample, it might be the case that successful water resources and water hazard predictions might not require anything that looks even like a simple hydrology model. This is all speculative, but the point is that the idea about hydrological understanding being necessary for reliable forecasting discussed in Section 5 may not be true even in the most superficial sense. This is an extreme and hypothetical example, but one that is worth (1) trying experimentally, and (2) being aware of as we calibrate our expectations about the role of hydrological theory and hydrological science in the context of big data and machine learning.

\subsection{Observations and Benchmarks}

Beven (2006b) proposed that the search for closure schemes at the watershed scale is the second most important problem in the discipline, with the most important being to improve observation capabilities. We agree completely. As was the case in 1987, the first and foremost job of hydrologists are and will continue to be related to improving observational capacity. The approaches discussed in this article only increase the need for observation data related to as many aspects of the water cycle as possible.

Shen et al. (2018) noted that past progress in the field of machine learning can be partially attributed to the culture of using public data sets and benchmarking new methods against previous state-of-the-art. There have been calls for consistent practices related to hypothesis testing, model intercomparison, and model rejection (e.g., Beven, 2018). While some of the philosophical counter-arguments to this are compelling (e.g., Baker, 2017; Nearing et al., 2020; Nearing, Tian, et al., 2016), without some community standard for benchmarking it is difficult to track progress in the field in an objective way.

This means that we need two things. First are better centralized data repositories. The community is aware of this (H. Gupta et al., 2014; Shen et al., 2018) and there are several such efforts happening in the field right now (e.g., Addor et al., 2017; Hoffman, Riley, Randerson, Keppel-Aleks, \& Lawrence, 2016; Newman et al., 2015). We expect that this issue will sort itself out in the near future. Still, our opinion is that one of the best investments that could be made in the discipline right now is to develop standardized and easily accessible big data repositories. The second thing we need is the willingness to use those data repositories. Just like in previous decades when the community 
responded to calls for making uncertainty quantification required for every modeling study (Pappenberger \& Beven, 2006), we need a community standard that requires all new modeling papers to include large-scale benchmarking against standard, centralized data sets.

Hydrological modeling is currently a field of ivory towers where legacy and affiliation guide the choice of model Addor and Melsen (2019) as opposed to empirical rigor Beven (2018). Different modeling groups largely work on their own models, and while there have been ad hoc intercomparisons (e.g., Best et al., 2015; van den Hurk et al., 2011), this is not routine and the hydrology community does not keep a list of current performance scores on standard test problems, as is standard in other communities (e.g., CMIP, ML-Perf, etc.).

\section{A White Whale}

During the community contribution phase of the IAHS 'Unsolved Problems in Hydrology' effort (Blöschl et al., 2019), one of the suggested questions was: "Does Machine Learning have a real role in hydrological modeling?" In contrast, we suggest that the existential question for our discipline right now is: "What role will hydrological science play in the age of machine learning?" van den Hurk et al. (2011) challenged that "it must be demonstrated that the model physics actually adds information to the prediction system." This is exactly the question that needs to be answered in order to understand how and where hydrological theory has a role to play in a world dominated by data. We see at least potential for deep learning to help address this by allowing us to decouple different parts of hydrological theory while still retaining scale-relevant predictive systems learned (partially) from data.

Very likely, the future of hydrology will be a mix of AI and physics-based approaches, but we have a hard time envisioning a future where transformative data science approaches like DL become simply another tool in the hydrologist's toolbox. We see it as much more likely that hydrological domain knowledge will become an integral part of guiding and developing fundamentally AI-based systems and analyses (e.g., Section 7.2).

Hydrology has roots - at least in part - as a branch of civil engineering. Klemeš (1986a) argued that "practices of bad science in hydrology cannot be blamed on engineers and other decision makers who 'need numbers.' For if these numbers are not to be based on sound hydrologic science but only on manipulations of arbitrary assumptions and concepts, hydrologists are not needed. Engineers can do such a job much better themselves since they at least can tailor the assumptions to the particular projects and, not mistaking them for scientific truth, will treat them accordingly in the decision process." The situation has not changed much in the 34 years since this was written: our ability to extract numbers (predictions) from data is advancing rapidly, but we have not improved very much our ability to make predictions from anything resembling hydrologic theory. While our models become increasingly complex, a well-calibrated Sacramento model is still one of the best in discipline.

The reason that we think this is an existential challenge is because we see hydrological science becoming increasingly decoupled from state-of-the-art hydrological information systems. Major development groups at governmental institutions internationally continue to dedicate the large majority of effort to the traditional models that have never benchmarked well against ML (e.g., Abramowitz, 2005; Best et al., 2015; Kratzert, Klotz, Herrnegger, et al., 2019; Nearing, Ruddell, et al., 2018). As far as we can tell, these models are dead on arrival. Barring some major fundamental theoretical discovery or innovation, there is essentially no chance that any incremental advancements will allow these models to catch up to the state-of-the-art hydrological predictions. Simultaneously, there has not been any serious or systematic investment into AI-based hydrology at a meaningful scale, and from what we can see (e.g., see Section 5) there is still strong re- 
sistance in the hydrology community toward adopting these approaches in a serious and fundamental way. Coupled with the fact that DL experiments demonstrate that hydrologists lack even a basic understanding of why their models fail (Section 2), this causes us to worry.

Our fear is that if the hydrological sciences community refuses to make a serious investment into the technology that works, then someone else will. This will mean a further decoupling between hydrological science (such as it is) and the societal value that this science is supposed to support. To be clear, the current authors do not want to see that happen, but we are not impressed with the reaction we are seeing in the community. Our message in this opinion piece is to stop assuming that the world needs our theories and expertise, and start demonstrating - quantitatively and systematically - the value of individual components of that expertise against the backdrop of a growing importance of big data.

Data Availability Statement: No data was generated as part of this project.

\section{Acknowledgments}

Authors from the Johannes Kepler University were partially supported by a Google faculty research award. Grey Nearing at the University of Alabama was supported by the NASA Advanced Information Systems Technology program (award ID 80NSSC17K0541). Jonathan Frame at the University of Alabama was supported by the NASA Terrestrial Hydrology Program (award ID 80NSSC18K0982). The author from IHCantabria acknowledges the financial support from the Government of Cantabria through the Fénix Programme.

\section{References}

Abramowitz, G. (2005). Towards a benchmark for land surface models. Geophysical Research Letters, 32(22).

Addor, N., \& Melsen, L. (2019). Legacy, rather than adequacy, drives the selection of hydrological models. Water Resources Research, 55(1), 378-390.

Addor, N., Newman, A. J., Mizukami, N., \& Clark, M. P. (2017). The camels data set: catchment attributes and meteorology for large-sample studies. Hydrology and Earth System Sciences (HESS), 21(10), 5293-5313.

Baker, V. R. (2017). Debates - hypothesis testing in hydrology: Pursuing certainty versus pursuing uberty. Water Resources Research, 53(3), 1770-1778.

Baldocchi, D., Falge, E., Gu, L., Olson, R., Hollinger, D., Running, S., .. o others (2001). Fluxnet: A new tool to study the temporal and spatial variability of ecosystem-scale carbon dioxide, water vapor, and energy flux densities. Bulletin of the American Meteorological Society, 82(11), 2415-2434.

Best, M. J., Abramowitz, G., Johnson, H., Pitman, A., Balsamo, G., Boone, A., ... others (2015). The plumbing of land surface models: benchmarking model performance. Journal of Hydrometeorology, 16(3), 1425-1442.

Beucler, T., Pritchard, M., Rasp, S., Gentine, P., Ott, J., \& Baldi, P. (2019). Enforcing analytic constraints in neural-networks emulating physical systems. arXiv preprint arXiv:1909.00912.

Beven, K. (1987). Towards a new paradigm in hydrology. IN: Water for the Future: Hydrology in Perspective. IAHS Publication(164).

Beven, K. (2000). Uniqueness of place and process representations in hydrological modelling.

Beven, K. (2006a). On undermining the science? Hydrological Processes: An International Journal, 20(14), 3141-3146.

Beven, K. (2006b). Searching for the holy grail of scientific hydrology: $\mathrm{Q} t=(\mathrm{s}, \mathrm{r}, \delta \mathrm{t})$ a as closure. Hydrology and Earth System Sciences, 10(5), 609-618. 
Beven, K. (2009). Comment on "equifinality of formal (dream) and informal (glue) bayesian approaches in hydrologic modeling?" by jasper a. vrugt, cajo jf ter braak, h gupta and bruce a. robinson. Stochastic environmental research and risk assessment, 23(7), 1059-1060.

Beven, K. (2016). Facets of uncertainty: epistemic uncertainty, non-stationarity, likelihood, hypothesis testing, and communication. Hydrological Sciences Journal, $61(9), 1652-1665$.

Beven, K. (2018). On hypothesis testing in hydrology: Why falsification of models is still a really good idea. Wiley Interdisciplinary Reviews: Water, 5(3), e1278.

Beven, K., \& Binley, A. (2014). Glue: 20 years on. Hydrological processes, 28(24), 5897-5918.

Beven, K., Smith, P., \& Freer, J. (2007). C Comment on "hydrological forecasting uncertainty assessment: Incoherence of the glue methodology" by pietro mantovan and ezio todini. Journal of Hydrology, 338(3-4), 315-318.

Beven, K., Smith, P., Westerberg, I., \& Freer, J. (2012). Comment on "pursuing the method of multiple working hypotheses for hydrological modeling" by p. clark et al. Water Resources Research, $48(11)$.

Beven, K., Smith, P., \& Wood, A. (2011). On the colour and spin of epistemic error (and what we might do about it). Hydrology and Earth System Sciences, 15, 3123-3133.

Beven, K., Smith, P. J., \& Freer, J. E. (2008). So just why would a modeller choose to be incoherent? Journal of hydrology, 354 (1-4), 15-32.

Bianchi, F., Rossiello, G., Costabello, L., Palmonari, M., \& Minervini, P. (2020). Knowledge graph embeddings and explainable ai. arXiv preprint arXiv:2004.14843.

Bishop, C. M. (1994). Mixture density networks.

Blöschl, G., Bierkens, M. F., Chambel, A., Cudennec, C., Destouni, G., Fiori, A., ... others (2019). Twenty-three unsolved problems in hydrology (uph)-a community perspective. Hydrological Sciences Journal, 64(10), 1141-1158.

Blöschl, G., \& Sivapalan, M. (1995). Scale issues in hydrological modelling: a review. Hydrological processes, 9(3-4), 251-290.

Chen, C., He, W., Zhou, H., Xue, Y., \& Zhu, M. (2020). A comparative study among machine learning and numerical models for simulating groundwater dynamics in the heihe river basin, northwestern china. Scientific Reports, 10(1), $1-13$.

Clark, M., Kavetski, D., \& Fenicia, F. (2011). Pursuing the method of multiple working hypotheses for hydrological modeling. Water Resources Research, $47(9)$.

Clark, M., Wilby, R. L., Gutmann, E. D., Vano, J. A., Gangopadhyay, S., Wood, A. W., ... Brekke, L. D. (2016). Characterizing uncertainty of the hydrologic impacts of climate change. Current Climate Change Reports, 2(2), 55-64.

Crawford, N. H., \& Burges, S. J. (2004). History of the stanford watershed model. Water Resour. Impact, 6(2), 1-3.

Daw, A., Thomas, R. Q., Carey, C. C., Read, J. S., Appling, A. P., \& Karpatne, A. (2019). Physics-guided architecture (pga) of neural networks for quantifying uncertainty in lake temperature modeling. arXiv preprint arXiv:1911.02682.

Daw, A., Thomas, R. Q., Carey, C. C., Read, J. S., Appling, A. P., \& Karpatne, A. (2020). Physics-guided architecture (pga) of neural networks for quantifying uncertainty in lake temperature modeling. In Proceedings of the 2020 siam international conference on data mining (pp. 532-540).

de Graaf, I., Condon, L., \& Maxwell, R. (2020). Hyper-resolution continental-scale 3 -d aquifer parameterization for groundwater modeling. Water Resources Research, 56 (5), e2019WR026004.

Dooge, J. C. (1986). Looking for hydrologic laws. Water Resources Research, $22(9 \mathrm{~S}), 46 \mathrm{~S}-58 \mathrm{~S}$. 
Einstein, A. (1926). The cause of the formation of meanders in the courses of rivers and of the so-called baer's law. Die Naturwissenschaften, 14 .

Einstein, H. A. (1950). The bed-load function for sediment transportation in open channel flows (Vol. 1026; Tech. Rep.). United States Department of Agriculture.

Entekhabi, D., Njoku, E. G., O’Neill, P. E., Kellogg, K. H., Crow, W. T., Edelstein, W. N., ... others (2010). The soil moisture active passive (smap) mission. Proceedings of the IEEE, 98(5), 704-716.

Fang, K., Pan, M., \& Shen, C. (2018). The value of smap for long-term soil moisture estimation with the help of deep learning. IEEE Transactions on Geoscience and Remote Sensing, 57(4), 2221-2233.

Fang, K., \& Shen, C. (2020). Near-real-time forecast of satellite-based soil moisture using long short-term memory with an adaptive data integration kernel. Journal of Hydrometeorology (2020).

Feng, D., Fang, K., \& Shen, C. (2019). Enhancing streamflow forecast and extracting insights using long-short term memory networks with data integration at continental scales.

Gauch, M., Mai, J., \& Lin, J. (2019). The proper care and feeding of camels: How limited training data affects streamflow prediction.

Gentine, P., Pritchard, M., Rasp, S., Reinaudi, G., \& Yacalis, G. ～(2018). Could machine learning break the convection parameterization deadlock? Geophysical Research Letters, 45(11), 5742-5751.

Ghahramani, Z., \& Roweis, S. T. (1999). Learning nonlinear dynamical systems using an em algorithm. In Advances in neural information processing systems (pp. 431-437).

Gould, S. J. (1999). Non-overlapping magisteria. Skeptical Inquirer, 23, 55-61.

Gupta, H., Perrin, C., Bloschl, G., Montanari, A., Kumar, R., Clark, M., \& Andréassian, V. (2014). Large-sample hydrology: a need to balance depth with breadth.

Gupta, H. V., Wagener, T., \& Liu, Y. (2008). Reconciling theory with observations: elements of a diagnostic approach to model evaluation. Hydrological Processes: An International Journal, 22(18), 3802-3813.

Hochreiter, S. (1991). Untersuchungen zu dynamischen neuronalen netzen (Unpublished doctoral dissertation). Technische Universität München.

Hochreiter, S., \& Schmidhuber, J. (1997). Long short-term memory. Neural Computation, 9(8), 1735-1780. Retrieved from https://doi.org/10.1162/neco 1997.9.8.1735 doi: 10.1162/neco.1997.9.8.1735

Hoffman, F. M., Riley, W. J., Randerson, J. T., Keppel-Aleks, G., \& Lawrence, D. M. (2016). International land model benchmarking (ilamb).

Hrachowitz, M., Savenije, H., Blöschl, G., McDonnell, J., Sivapalan, M., Pomeroy, J., ... others (2013). A decade of predictions in ungauged basins (pub) - a review. Hydrological sciences journal, 58(6), 1198-1255.

Hsu, K.-l., Gupta, H. V., \& Sorooshian, S. (1995). Artificial neural network modeling of the rainfall-runoff process. Water resources research, 31(10), 25172530 .

Jiang, S., Zheng, Y., \& Solomatine, D. (2020). Improving ai system awareness of geoscience knowledge: Symbiotic integration of physical approaches and deep learning. Geophysical Research Letters, 47(13), e2020GL088229.

Karpatne, A., Atluri, G., Faghmous, J. H., Steinbach, M., Banerjee, A., Ganguly, A., ... Kumar, V. (2017). Theory-guided data science: A new paradigm for scientific discovery from data. IEEE Transactions on Knowledge and Data Engineering, 29(10), 2318-2331.

Kirchner, J. W. (2006). Getting the right answers for the right reasons: Linking measurements, analyses, and models to advance the science of hydrology. $W a-$ ter Resources Research, 42(3). 
Klemeš, V. (1986a). Dilettantism in hydrology: Transition or destiny? Water Resources Research, 22 (9S), 177S-188S.

Klemeš, V. (1986b). Operational testing of hydrological simulation models. Hydrological Sciences Journal, 31(1), 13-24.

Kolassa, J., Reichle, R., Liu, Q., Alemohammad, S., Gentine, P., Aida, K., .. others (2018). Estimating surface soil moisture from smap observations using a neural network technique. Remote sensing of environment, 204, 43-59.

Kratzert, F., Herrnegger, M., Klotz, D., Hochreiter, S., \& Klambauer, G. (2019). Neuralhydrology-interpreting lstms in hydrology. In Explainable ai: Interpreting, explaining and visualizing deep learning (pp. 347-362). Springer.

Kratzert, F., Klotz, D., Herrnegger, M., Sampson, A. K., Hochreiter, S., \& Nearing, G. (2019). Toward improved predictions in ungauged basins: Exploiting the power of machine learning. Water Resources Research.

Kratzert, F., Klotz, D., Hochreiter, S., \& Nearing, G. (2020). A note on leveraging synergy in multiple meteorological datasets with deep learning for rainfallrunoff modeling. Hydrology and Earth System Sciences Discussions.

Kratzert, F., Klotz, D., Shalev, G., Klambauer, G., Hochreiter, S., \& Nearing, G. (2019). Towards learning universal, regional, and local hydrological behaviors via machine learning applied to large-sample datasets. Hydrology and Earth System Sciences, 23(12), 5089-5110.

Kuhn, T. S. (1962). The structure of scientific revolutions. University of Chicago press.

Kumar, P. (2011). Typology of hydrologic predictability. Water Resources Research, $47(3)$.

Kumar, S. V., Reichle, R. H., Harrison, K. W., Peters-Lidard, C. D., Yatheendradas, S., \& Santanello, J. A. (2012). A comparison of methods for a priori bias correction in soil moisture data assimilation. Water Resources Research, 48(3).

Laudan, L. (1990). Demystifying underdetermination. Minnesota studies in the philosophy of science, 14(1990), 267-297.

Li, H.-Y., Leung, L. R., Getirana, A., Huang, M., Wu, H., Xu, Y., ... Voisin, N. (2015). Evaluating global streamflow simulations by a physically based routing model coupled with the community land model. Journal of Hydrometeorology, 16(2), 948-971.

Lin, P., Pan, M., Beck, H. E., Yang, Y., Yamazaki, D., Frasson, R., ... others (2019). Global reconstruction of naturalized river flows at 2.94 million reaches. Water resources research, 55(8), 6499-6516.

Mantovan, P., \& Todini, E. (2006). Hydrological forecasting uncertainty assessment: Incoherence of the glue methodology. Journal of hydrology, 330(1-2), 368-381.

Martinez, G. F., \& Gupta, H. V. (2010). Toward improved identification of hydrological models: A diagnostic evaluation of the "abcd" monthly water balance model for the conterminous united states. Water Resources Research, 46(8).

McDonnell, J., Sivapalan, M., Vaché, K., Dunn, S., Grant, G., Haggerty, R., ... others (2007). Moving beyond heterogeneity and process complexity: A new vision for watershed hydrology. Water Resources Research, 43(7).

Mo, S., Zabaras, N., Shi, X., \& Wu, J. (2019). Deep autoregressive neural networks for high-dimensional inverse problems in groundwater contaminant source identification. Water Resources Research, 55(5), 3856-3881.

Montanari, A. (2007). What do we mean by 'uncertainty'? the need for a consistent wording about uncertainty assessment in hydrology. Hydrological Processes: An International Journal, 21(6), 841-845.

Montanari, A., \& Koutsoyiannis, D. (2012). A blueprint for process-based modeling of uncertain hydrological systems. Water Resources Research, 48(9).

Moshe, Z., Metzger, A. F., Kratzert, F., Elidan, G., Nevo, S., \& El-Yaniv, R. (2020). Hydronets: Leveraging river structure for hydrologic modeling.

Nabian, M. A., \& Meidani, H. (2020). Physics-driven regularization of deep neural 
networks for enhanced engineering design and analysis. Journal of Computing and Information Science in Engineering, 20(1).

Nagel, E. (1961). The structure of science.

Nearing, G. (2013). Diagnostics and generalizations for parametric state estimation.

Nearing, G. (2014). Comment on "a blueprint for process-based modeling of uncertain hydrological systems" by alberto montanari and demetris kousoyiannis.

Nearing, G., \& Gupta, H. (2015). The quantity and quality of information in hydrologic models. Water Resources Research, 51(1), 524-538.

Nearing, G., \& Gupta, H. (2018). Ensembles vs. information theory: supporting science under uncertainty. Frontiers of Earth Science, 12(4), 653-660.

Nearing, G., Gupta, H., \& Crow, W. (2013). Information loss in approximately bayesian estimation techniques: A comparison of generative and discriminative approaches to estimating agricultural productivity. Journal of hydrology, 507, $163-173$.

Nearing, G., Mocko, D. M., Peters-Lidard, C. D., Kumar, S. V., \& Xia, Y. (2016). Benchmarking nldas-2 soil moisture and evapotranspiration to separate uncertainty contributions. Journal of hydrometeorology, 17(3), 745-759.

Nearing, G., Ruddell, B., Bennett, A., Prieto, C., \& Gupta, H. (2020). Debates: Does information theory provide a new paradigm for earth science? hypothesis testing. Water Resources Research.

Nearing, G., Ruddell, B. L., Clark, M. P., Nijssen, B., \& Peters-Lidard, C. (2018). Benchmarking and process diagnostics of land models. Journal of Hydrometeorology, 19(11), 1835-1852.

Nearing, G., Tian, Y., Gupta, H., Clark, M., Harrison, K., \& Weijs, S. (2016). A philosophical basis for hydrological uncertainty. Hydrological Sciences Journal, $61(9), 1666-1678$.

Nearing, G., Yatheendradas, S., Crow, W., Zhan, X., Liu, J., \& Chen, F. (2018). The efficiency of data assimilation. Water resources research, 54 (9), 63746392.

Newman, A., Clark, M., Sampson, K., Wood, A., Hay, L., Bock, A., ... others (2015). Development of a large-sample watershed-scale hydrometeorological data set for the contiguous usa: data set characteristics and assessment of regional variability in hydrologic model performance. Hydrology and Earth System Sciences, 19(1), 209-223.

Pappenberger, F., \& Beven, K. (2006). Ignorance is bliss: Or seven reasons not to use uncertainty analysis. Water resources research, 42(5).

Parry, R. (2003). Episteme and techne.

Pearl, J. (2013). Structural counterfactuals: A brief introduction. Cognitive science, $37(6), 977-985$.

Pelissier, C., Frame, J., \& Nearing, G. (2020). Combining parametric land surface models with machine learning. arXiv preprint arXiv:2002.06141.

Peters-Lidard, C. D., Clark, M., Samaniego, L., Verhoest, N. E., Van Emmerik, T., Uijlenhoet, R., .. Woods, R. (2017). Scaling, similarity, and the fourth paradigm for hydrology. Hydrology and earth system sciences, 21 (7), 3701.

Rackauckas, C., Ma, Y., Martensen, J., Warner, C., Zubov, K., Supekar, R., ... Ramadhan, A. (2020). Universal differential equations for scientific machine learning. arXiv preprint arXiv:2001.04385.

Renard, B., Kavetski, D., Kuczera, G., Thyer, M., \& Franks, S. W. (2010). Understanding predictive uncertainty in hydrologic modeling: The challenge of identifying input and structural errors. Water Resources Research, 46(5).

Ribeiro, M. T., Singh, S., \& Guestrin, C. (2016). Model-agnostic interpretability of machine learning. arXiv preprint arXiv:1606.05386.

Rodell, M., Houser, P., Jambor, U., Gottschalck, J., Mitchell, K., Meng, C.-J., ... others (2004). The global land data assimilation system. Bulletin of the American Meteorological Society, 85(3), 381-394. 
Ruddell, B. L., Drewry, D. T., \& Nearing, G. (2019). Information theory for model diagnostics: Structural error is indicated by trade-off between functional and predictive performance. Water Resources Research, 55 (8), 6534-6554.

Sahoo, S., Russo, T. A., Elliott, J., \& Foster, I. (2017). Machine learning algorithms for modeling groundwater level changes in agricultural regions of the us. Water Resources Research, 53(5), 3878-3895.

Salas, F. R., Somos-Valenzuela, M. A., Dugger, A., Maidment, D. R., Gochis, D. J., David, C. H., ... Noman, N. (2018). Towards real-time continental scale streamflow simulation in continuous and discrete space. JAWRA Journal of the American Water Resources Association, 54(1), 7-27.

Samek, W. (2019). Explainable ai: interpreting, explaining and visualizing deep learning (Vol. 11700). Springer Nature.

Schaefer, G. L., Cosh, M. H., \& Jackson, T. J. (2007). The usda natural resources conservation service soil climate analysis network (scan). Journal of Atmospheric and Oceanic Technology, 24(12), 2073-2077.

Sellars, S. (2018). "grand challenges" in big data and the earth sciences. Bulletin of the American Meteorological Society, 99(6), ES95-ES98.

Shen, C., Laloy, E., Elshorbagy, A., Albert, A., Bales, J., Chang, F.-J., ... Tsai, W.-P. (2018). Hess opinions: Incubating deep-learning-powered hydrologic science advances as a community. Hydrology and Earth System Sciences(11), 5639-5656. doi: 10.5194/hess-22-5639-2018

Singh, V. P., Yang, C. T., \& Deng, Z. (2003). Downstream hydraulic geometry relations: 1. theoretical development. Water Resources Research, 39(12).

Sivapalan, M. (2006). Pattern, process and function: elements of a unified theory of hydrology at the catchment scale. Encyclopedia of hydrological sciences.

Sivapalan, M., Takeuchi, K., Franks, S., Gupta, V., Karambiri, H., Lakshmi, V., ... others (2003). Iahs decade on predictions in ungauged basins (pub), 20032012: Shaping an exciting future for the hydrological sciences. Hydrological sciences journal, 48(6), 857-880.

Stedinger, J. R., Vogel, R. M., Lee, S. U., \& Batchelder, R. (2008). Appraisal of the generalized likelihood uncertainty estimation (glue) method. Water resources research, $44(12)$.

Sundararajan, M., Taly, A., \& Yan, Q. (2017). Axiomatic attribution for deep networks. In Proceedings of the 34th international conference on machine learning-volume 70 (pp. 3319-3328).

Taylor, J. W. (2000). A quantile regression neural network approach to estimating the conditional density of multiperiod returns. Journal of Forecasting, 19(4), 299-311.

Todini, E. (2007). Hydrological catchment modelling: past, present and future. $H y$ drology and Earth System Sciences, 11(1), 468-482.

Todini, E., \& Mantovan, P. (2007). Comment on:'on undermining the science?'by keith beven. Hydrological Processes: An International Journal, 21 (12), 16331638 .

van den Hurk, B., Best, M., Dirmeyer, P., Pitman, A., Polcher, J., \& Santanello, J. (2011). Acceleration of land surface model development over a decade of glass. Bulletin of the American Meteorological Society, 92(12), 1593-1600.

Vrugt, J. A., Ter Braak, C. J., Gupta, H., \& Robinson, B. A. (2009). Equifinality of formal (dream) and informal (glue) bayesian approaches in hydrologic modeling? Stochastic environmental research and risk assessment, 23(7), 1011-1026.

Wagener, T., Sivapalan, M., Troch, P., \& Woods, R. (2007). Catchment classification and hydrologic similarity. Geography compass, 1(4), 901-931.

Wang, J., \& Bras, R. (2011). A model of evapotranspiration based on the theory of maximum entropy production. Water Resources Research, $47(3)$.

Wang, Q., Mao, Z., Wang, B., \& Guo, L. (2017). Knowledge graph embedding: A 
survey of approaches and applications. IEEE Transactions on Knowledge and Data Engineering, 29(12), 2724-2743.

Weinberg, G. M. (1975). An introduction to general systems thinking (Vol. 304). Wiley New York.

Williams, C. K., \& Rasmussen, C. E. (2006). Gaussian processes for machine learning (Vol. 2) (No. 3). MIT press Cambridge, MA.

Wood, E. F., Roundy, J. K., Troy, T. J., Van Beek, L., Bierkens, M. F., Blyth, E., ... others (2011). Hyperresolution global land surface modeling: Meeting a grand challenge for monitoring earth's terrestrial water. Water Resources Research, 47(5).

Yilmaz, K. K., Gupta, H. V., \& Wagener, T. (2008). A process-based diagnostic approach to model evaluation: Application to the nws distributed hydrologic model. Water Resources Research, $44(9)$. 